PROCEEDINGS OF THE

AMERICAN MATHEMATICAL SOCIETY

Volume 139, Number 1, January 2011, Pages 273-282

S 0002-9939(2010)10516-6

Article electronically published on July 12, 2010

\title{
FAR-FROM-EXPIRY BEHAVIOR OF THE AMERICAN PUT OPTION ON A DIVIDEND-PAYING ASSET
}

\author{
XINFU CHEN, HUIBIN CHENG, AND JOHN CHADAM \\ (Communicated by Walter Craig)
}

\begin{abstract}
We provide a rigorous proof of sharp estimates for the long time behavior of the early exercise boundary and the price for an American put option on a dividend-paying asset that follows a geometric Brownian motion.
\end{abstract}

\section{INTRODUCTION}

Recently Ahn et al. 1 announced a proof of the following long time asymptotic behavior of the infinite horizon (Merton) problem for the American put option on a zero dividend asset as the scaled time-to-expiry $s:=\frac{\sigma^{2}}{2}(T-t) \rightarrow \infty$ :

$$
\begin{gathered}
b(s)=b^{*}+o(1) e^{-(k+1)^{2} s / 4} \\
\left\|p(\cdot, s)-p^{*}(\cdot)\right\|_{L^{\infty}(\mathbb{R})}=O(1) \begin{cases}s^{-1 / 2} e^{-k s} & \text { if } 0<k<1, \\
e^{-(k+1)^{2} s / 4} & \text { if } k \geqslant 1,\end{cases}
\end{gathered}
$$

where $k=2 r \sigma^{-2}$ ( $r$ is the risk-free interest rate and $\sigma$ is the volatility of the asset that follows a geometric Brownian motion) and $\left(b^{*}, p^{*}(\cdot)\right)$ is the Merton solution for the infinite horizon problem. In [2] we provided the outline of the proof of a stronger result:

$$
b(s)=b^{*}+[m+o(1)] s^{-3 / 2} e^{-(k+1)^{2} s / 4} \quad \text { as } s \rightarrow \infty
$$

where $m$ is a positive constant that can be easily determined numerically. In this paper, we follow the steps outlined in 2 to provide a proof of our sharper result in the more general setting of a dividend-paying asset (i.e., for all dividend rates $D \geqslant 0)$. We also provide generalizations of (1.2) for arbitrary $D \geqslant 0$. The precise statements of these results are given in Theorems 13 in the next section. The proofs capture the changes in the estimates arising from the variation in $D$. Moreover, our proofs do not require the convexity of the free boundary in contrast with the results in [1] where it plays a crucial role. This observation is especially significant since

Received by the editors June 1, 2009 and, in revised form, December 22, 2009 and March 3, 2010 .

2010 Mathematics Subject Classification. Primary 35R35, 91G20, 91G80.

The first author acknowledges support from NSF grant DMS-0504691.

The second and third authors acknowledge support from NSF grant DMS-0707953.

The authors would like to thank the referees for their comments, which have improved the presentation of the results. 
we have recently provided a rigorous proof [3] that the early exercise boundary is not convex when $0<D-r \ll 1$.

Finally, we mention that the results obtained here are of practical as well as mathematical interest. As demonstrated in [2, the far-from-expiry behavior can be interpolated with the near-expiry behavior to produce simple analytic formulae that approximate the early exercise boundary and price of the American put uniformly for any time from expiry with amazing accuracy. On the mathematical side, an open problem that has attracted increasing interest recently is the question of the convexity of the boundary, especially for $D>0$. Specifically it has been shown rigorously that the boundary is convex when $D=0$ (cf. references in [3]) and, as mentioned earlier, not convex for $0<D-r \ll 1$ with the non-convex region occurring close to expiry. Numerical evidence suggests that for all other cases the boundary is convex. We anticipate that these precise far-from-expiry estimates will be useful in proving that for all $D \geq 0$ the boundary is convex sufficiently far from expiry.

\section{MAin RESULTS}

We consider a financial market consisting of a money account and a stock, whose time $t$ prices, $\mathbf{B}_{t}$ and $\mathbf{S}_{t}$, are stochastic processes defined by the stochastic differential equations

$$
d \mathbf{S}_{t}=\mu_{t} \mathbf{S}_{t} d t+\sigma \mathbf{S}_{t} d \mathbf{W}_{t}, \quad d \mathbf{B}_{t}=r \mathbf{B}_{t} d t
$$

where $\sigma$ and $r$ are positive constants and $\left\{\mathbf{W}_{t}\right\}$ is the standard Brownian motion (Wiener process). In the time interval $[t, t+d t)$, the stock pays $D \mathbf{S}_{t} d t$ dividend at time $t+d t$. An American put option with strike price $E$ and expiry $T$ is a guaranteed right to sell a stock at price $E$ at any time on or before expiry. It follows from the Black-Scholes theory (cf. [3]; please see the references in [1, 2, 3] for the standard finance terminology used in this note) that the no-arbitrage price of the option at time $t$ is $P\left(\mathbf{S}_{t}, t\right)$ and the optimal exercise time is $\tau^{*}:=\sup \{t \leqslant$ $\left.T \mid \mathbf{S}_{s}>B(s) \forall s \in\left[t_{0}, t\right)\right\}$, where $t_{0}$ is the current time and $(B, P)$ is the classical solution of the variational inequality

$$
\begin{cases}\max \left\{\mathcal{L}^{*} P,(E-S)^{+}-P\right\}=0 & \text { in }(0, \infty) \times(-\infty, T), \\ P(S, T)=(E-S)^{+}:=\max \{E-S, 0\} & \text { on }(0, \infty) \times\{T\}, \\ B(t):=\inf \left\{S>0 \mid P(S, t)>(E-S)^{+}\right\} & \text {on }(-\infty, T]\end{cases}
$$

where $\mathcal{L}^{*} P=\frac{\partial P}{\partial t}+\frac{\sigma^{2}}{2} S^{2} \frac{\partial^{2} P}{\partial S^{2}}+(r-D) S \frac{\partial P}{\partial S}-r P$. Using the dimensionless quantities,

$$
\begin{gathered}
x:=\ln (S / E), \quad s:=(T-t) \sigma^{2} / 2, \quad p(x, s):=P(S, t) / E, \quad b(s):=\ln (B(t) / E), \\
k:=2 r \sigma^{-2}, \quad \ell:=2 D \sigma^{-2}, \quad \alpha:=k-\ell-1, \quad \beta:=k+\alpha^{2} / 4, \\
p_{0}(x):=\max \left\{1-e^{x}, 0\right\}, \quad \mathcal{L} p:=p_{x x}+\alpha p_{x}-k p,
\end{gathered}
$$

the variational problem for $(B, P)$ is transformed to

$$
\left\{\begin{array}{l}
\max \left\{\mathcal{L} p-p_{s}, p_{0}-p\right\}=0 \text { in } \mathbb{R} \times(0, \infty), \quad p(\cdot, 0)=p_{0} \\
b(s):=\inf \left\{x \mid p(x, s)>p_{0}(x)\right\} \quad \forall s>0
\end{array}\right.
$$


We are interested in the behavior of $b(s)$ and $p(x, s)$ as $s \rightarrow \infty$. By a comparison argument, we have

$$
p(\cdot, s) \nearrow p^{*}(\cdot), \quad b(s) \searrow b^{*} \quad \text { as } s \nearrow \infty,
$$

where $\left(p^{*}, b^{*}\right)$ is the solution of the infinite horizon problem, given by

$$
p^{*}(x):=\max \left\{1-e^{x}, \frac{e^{-\lambda\left(x-b^{*}\right)}}{1+\lambda}\right\}, \quad b^{*}:=\ln \frac{\lambda}{1+\lambda}, \quad \lambda:=\frac{\alpha}{2}+\sqrt{\beta} .
$$

In this short paper, we prove the following:

Theorem 1. There exists a constant $m>0$ such that for each $s \geqslant 1$,

$$
b(s)=b^{*}+\left[m+O(1) s^{-\frac{1}{2}}\right] s^{-\frac{3}{2}} e^{-\beta s}, \quad \dot{b}(s)=-\left[m \beta+O(1) s^{-\frac{1}{2}}\right] s^{-\frac{3}{2}} e^{-\beta s},
$$

where $O(1)$ is a generic function bounded uniformly in $s \in[1, \infty)$.

Theorem 2 (Decay Rates). There exist positive constants $c$ and $C$ such that for each $s \geqslant 1$,

$$
c \rho(s) \leqslant\left\|p(\cdot, s)-p^{*}(\cdot)\right\|_{L^{\infty}(\mathbb{R})} \leqslant C \rho(s), \quad c \rho(s) \leqslant\left\|p_{s}(\cdot, s)\right\|_{L^{\infty}(\mathbb{R})} \leqslant C \rho(s),
$$

where

$$
\rho(s)= \begin{cases}s^{-\frac{3}{2}} e^{-\beta s} & \text { if } \alpha>0 \\ s^{-1} e^{-k s} & \text { if } \alpha=0 \\ s^{-\frac{1}{2}} e^{-k s} & \text { if } \alpha<0\end{cases}
$$

Theorem 3 (Asymptotic Profiles). There exist constants $c_{1}>0$ and $c_{2} \in \mathbb{R}$ that depend only on $k$ and $\ell$ such that for every $x \in \mathbb{R}$ and $s \geqslant 2$,

$$
\frac{p_{s}(x, s)}{\rho(s)}= \begin{cases}e^{-\xi^{2}}\left\{c_{1}+c_{2} \xi s^{-\frac{1}{2}}+O(1)\left(1+\xi^{2}\right) s^{-1}\right\} & \text { if } \alpha<0, \\ z^{+} e^{-[z+b(s) / \sqrt{4 s}]^{2}}\left\{c_{1}+O(1)(1+z \ln s) s^{-1 / 2}\right\} & \text { if } \alpha=0, \\ {[x-b(s)]^{+} e^{-x^{2} /(4 s)-\alpha x / 2}\left\{c_{1}+O(1)\left(s^{-1 / 2}+|x| s^{-1} \ln s\right)\right\}} & \text { if } \alpha>0,\end{cases}
$$

where $\xi:=(x+\alpha s) / \sqrt{4 s}, z:=(x-b(s)) / \sqrt{4 s}, z^{+}:=\max \{0, z\}$, and $O(1)$ is a function bounded uniformly in $(x, s) \in \mathbb{R} \times[1, \infty)$. Consequently, for some positive constant $c$ depending on $k$ and $\ell$,

$$
\lim _{s \rightarrow \infty}\left\|\frac{p^{*}-p}{\rho(s)}-\Psi\right\|_{L^{\infty}(\mathbb{R})}=0 ; \quad \Psi(x, s):= \begin{cases}c e^{-\xi^{2}} & \text { if } \alpha<0, \\ c z^{+} e^{-z^{2}} & \text { if } \alpha=0, \\ c\left[x-b^{*}\right]^{+} e^{-\alpha x / 2} & \text { if } \alpha>0 .\end{cases}
$$

Proof of Theorem 1. In the case $\ell=0$ (i.e. no dividend), we have already provided an outline of the proof in [2]. Here we follow that outline and provide the full details for the general case $\ell \geqslant 0$.

In [3] we showed that

$b \in C^{\infty}((0, \infty)), \quad \lim _{s \searrow 0} b(s)=b_{0}:=\min \{0, \ln (k / \ell)\}, \quad \dot{b}(s)<0, \ell e^{b(s)}-k<0 \forall s>0$.

Moreover, in $\S 2$ of 2 we showed by passing to the limit from the approximate solution to (2.1) that $p \in W^{2, \infty}$ and $p(x, s)>p_{0}(x)$ for $x>b(s)$. Hence

$$
\mathcal{L} p-p_{s}= \begin{cases}\mathcal{L} p_{0} & \text { if } x<b(s) \\ 0 & \text { if } x>b(s)\end{cases}
$$


Denoting by $\Gamma$ the fundamental solution of $\partial_{s}-\mathcal{L}$,

$$
\Gamma(x, s):=(4 \pi s)^{-1 / 2} e^{-(x+\alpha s)^{2} /(4 s)-k s}=(4 \pi s)^{-1 / 2} e^{-x^{2} /(4 s)-\alpha x / 2-\beta s},
$$

and using Green's Theorem, one obtains

$$
p(x, s)=\int_{-\infty}^{\infty} \Gamma(x-y, s) p_{0}(y) d y+\int_{0}^{s} \int_{b(s-t)}^{\infty} \Gamma(x-y, t) \mathcal{L} p_{0}(y) d y d t, \forall(x, s) \in \mathbb{R} \times[0, \infty) .
$$

By differentiating (2.2), we obtain

$$
\begin{aligned}
p_{s}(x, s) & =\Gamma(x, s)+\int_{b_{0}}^{0}\left[\ell e^{y}-k\right] \Gamma(x-y, s) d y-\int_{0}^{s} \dot{b}(t)\left[\ell e^{b(t)}-k\right] \Gamma(x-b(t), s-t) d t \\
p_{s x}(x, s)= & \Gamma_{x}(x, s)+\int_{b_{0}}^{0}\left[\ell e^{y}-k\right] \Gamma_{x}(x-y, s) d y \\
& -\int_{0}^{s} \dot{b}(t)\left[\ell e^{b(t)}-k\right] \Gamma_{x}(x-b(t), s-t) d t .
\end{aligned}
$$

Evaluating these at $x=b(s)$ and noting that $p_{s}(b(s) \pm, s)=0$ and $p_{s x}(b(s) \pm, s)=$ $(1 / 2 \pm 1 / 2) \dot{b}(s)\left[\ell e^{b(s)}-k\right]$, we obtain

$$
\begin{aligned}
& 0= \Gamma(b(s), s)+\int_{b_{0}}^{0}\left[\ell e^{y}-k\right] \Gamma(b(s)-y, s) d y \\
&-\int_{0}^{s} \dot{b}(t)\left[\ell e^{b(t)}-k\right] \Gamma(b(s)-b(t), s-t) d t \\
& \dot{b}(s)\left[\ell e^{b(s)}-k\right]= 2 \Gamma_{x}(b(s), s)+2 \int_{b_{0}}^{0}\left[\ell e^{y}-k\right] \Gamma_{x}(b(s)-y, s) d y \\
&-2 \int_{0}^{t} \dot{b}(t)\left[\ell e^{b(t)}-k\right] \Gamma_{x}(b(s)-b(t), s-t) d t .
\end{aligned}
$$

Multiplying (2.3) by $b(s) / s+\alpha$ and adding it to (2.4) yields the equation

$$
\begin{aligned}
\dot{b}(s)\left[\ell e^{b(s)}-k\right] & =\frac{1}{s} \int_{b_{0}}^{0}\left[\ell e^{y}-k\right] y \Gamma(b(s)-y, s) d y \\
& -\int_{0}^{s} \dot{b}(t)\left[\ell e^{b(t)}-k\right]\left(\frac{b(s)}{s}-\frac{b(s)-b(t)}{s-t}\right) \Gamma(b(s)-b(t), s-t) d t,
\end{aligned}
$$

where we used $\Gamma_{x}(x, s)=-(x+\alpha s) / 2 s \Gamma(x, s)$. Dividing (2.3) and (2.5) by $\Gamma(b(s), s)$ and $\Gamma(b(s), s) / s$, respectively, we obtain the following integral identities that are valid for all $\ell \geq 0$ :

$$
\begin{gathered}
1+\int_{b_{0}}^{0} A(s, y) d y=\int_{0}^{s} B(s, t) d t \\
\frac{\dot{b}(s)\left[\ell e^{b(s)}-k\right] s}{\Gamma(b(s), s)}=\int_{b_{0}}^{0} y A(s, y) d y+\int_{0}^{s}[t \delta(s, t)-b(t)] B(s, t) d t,
\end{gathered}
$$


where, for $s>0, y \in\left(b_{0}, 0\right), t \in(0, s)$,

$$
\begin{aligned}
A(s, y) & :=\left[\ell e^{y}-k\right] \frac{\Gamma(b(s)-y, s)}{\Gamma(b(s), s)}=A_{\infty}(y) e^{\delta_{1}(s, y)}, \\
B(s, t) & :=\dot{b}(t)\left[\ell e^{b(t)}-k\right] \frac{\Gamma(b(s)-b(t), s-t)}{\Gamma(b(s), s)}=\zeta(t)\left(1-\frac{t}{s}\right)^{-1 / 2} e^{\delta_{2}(s, t)}, \\
A_{\infty}(y) & :=\left[\ell e^{y}-k\right] e^{\alpha y / 2}, \quad \zeta(t):=\dot{b}(t)\left[\ell e^{b(t)}-k\right] e^{\beta t+\alpha b(t) / 2}, \\
\delta(s, t) & :=\frac{b(s)-b(t)}{s-t}, \quad \delta_{1}(s, y):=\frac{2 b(s) y-y^{2}}{4 s}, \quad \delta_{2}(s, t):=\frac{b^{2}(s)}{4 s}-\frac{[b(s)-b(t)]^{2}}{4(s-t)} .
\end{aligned}
$$

Note that the terms $\int_{b_{0}}^{0} A d y$ and $\int_{b_{0}}^{0} y A d y$ do not appear in 2] since $b_{0}=0$ when $\ell \leqslant k$.

Observe that $A>0, B>0, \delta<0$. It then follows from (2.7) and (2.6) that for every $s \geqslant 1$,

$0<\frac{\dot{b}(s)\left[\ell e^{b(s)}-k\right] s}{\Gamma(b(s), s)} \leqslant|b(s)| \int_{0}^{s} B(s, t) d t=|b(s)|\left\{1+\int_{b_{0}}^{0} A(s, y) d y\right\} \leqslant\left|b^{*}\right| C_{1}$,

where $C_{1}=1+\left|b_{0}\right|(\ell-k) e^{\left|b_{0} \alpha\right| / 2+b_{0} b^{*} / 2}$. Hence, $\dot{b}(s)=O(1) s^{-3 / 2} e^{-\beta s}$ for $s \geqslant 1$.

Once we have the upper bound of $|\dot{b}|$, we can study the asymptotic behavior of the right-hand side of (2.7) as $s \rightarrow \infty$. First, for each $s \geqslant 1, t \in[0, s]$, and $y \in\left[b_{0}, 0\right]$,

$$
\delta(s, t)=O(1) s^{-1}, \quad \delta_{1}(s, y)=O(1) s^{-1}, \quad \delta_{2}(s, t)=O(1) s^{-1} .
$$

Next note that $\zeta(t)=O(1) t^{-3 / 2}$ for $t \in[1, \infty)$ and $\zeta(t)=O(1) \dot{b}(t)$ for $t \in[0,1]$. Hence, for $s \geqslant 1$,

$$
\begin{aligned}
\int_{s}^{\infty} \zeta(t) d t & =O(1) \int_{s}^{\infty} t^{-3 / 2} d t=O(1) s^{-1 / 2} \\
\int_{s / 2}^{s} \zeta(t)\left(1-\frac{t}{s}\right)^{-\frac{1}{2}} d t & =O(1) \int_{s / 2}^{s} \frac{t^{-3 / 2} \sqrt{s}}{\sqrt{s-t}} d t=\frac{O(1)}{\sqrt{s}} \\
\int_{0}^{s / 2} \frac{t+1}{s} \zeta(t) d t & =\frac{O(1)}{s} \int_{0}^{1 / 2} \dot{b}(t) d t+\frac{O(1)}{s} \int_{1 / 2}^{s / 2} t^{-1 / 2} d t=\frac{O(1)}{\sqrt{s}} .
\end{aligned}
$$

Thus, using $(1-t / s)^{-1 / 2}=1+O(1) t / s$ for $t \in[0, s / 2]$ we obtain from (2.7) that

$$
\frac{\dot{b}(s)\left[\ell e^{b(s)}-k\right] s}{\Gamma(b(s), s)}=m_{1}+\frac{O(1)}{\sqrt{s}} ; \quad m_{1}:=\int_{b_{0}}^{0} y A_{\infty}(y) d y-\int_{0}^{\infty} b(t) \zeta(t) d t .
$$

Since $b(s)=b^{*}+O(1) e^{-\beta s}$, this implies that $\dot{b}(s)=-\left[m \beta+O(1) s^{-1 / 2}\right] s^{-\frac{3}{2}} e^{-\beta s}$ and after integration, $b(s)=b^{*}+\left[m+O(1) s^{-1 / 2}\right] s^{-3 / 2} e^{-\beta s}$, where $m=m_{1} e^{-\alpha b^{*} / 2} /$ $\left(\sqrt{4 \pi}\left[k-\ell e^{b^{*}}\right] \beta\right)$. To see that $m$ is positive, we let $s \rightarrow \infty$ in (2.6) to obtain the identity $1+\int_{b_{0}}^{0} A_{\infty}(y) d y-\int_{0}^{\infty} \zeta(t) d t=0$. Adding a multiple of $-b_{0}$ of this identity to the defining equation of $m_{1}$ we find that

$$
m_{1}=-b_{0}+\int_{b_{0}}^{0}\left[y-b_{0}\right] A_{\infty}(y) d y+\int_{0}^{\infty}\left[b_{0}-b(t)\right] \zeta(t) d t>0 .
$$

This completes the proof of Theorem 1 .

Proof of Theorem 2, Note that the function $q(x, s):=p_{s}(x, s) e^{\alpha x / 2+\beta s}$ satisfies $q_{s}-q_{x x}=0 \quad$ in $Q_{b}:=\{(x, s) \mid x>b(s), s>0\}, \quad q(b(s), s)=0 \quad \forall s>0$. 
Since $p \geqslant p_{0}$ implies that $q(\cdot, 0)=p_{s}(\cdot, 0) \geqslant 0$, so by the maximum principle and Hopf's Lemma, $q>0$ in $Q_{b}$ (see [3, section 2] for the details of a rigorous derivation). We shall construct comparison functions to estimate the upper and lower bounds of $q$.

Upper bound. Fix an arbitrary $T>0$. Let $\bar{q}$ be the solution of

$$
\begin{gathered}
\bar{q}_{s}-\bar{q}_{x x}=0 \text { in }[b(T), \infty) \times(0, T], \bar{q}=0 \text { on }\{b(T)\} \times[0, T], \\
\bar{q}=q \text { on }[b(T), \infty) \times\{0\} .
\end{gathered}
$$

Then by comparison, $q \leqslant \bar{q}$ on $[b(T), \infty) \times[0, T]$. In particular, for $x \geqslant b(T)$,

$$
q(x, T) \leqslant \bar{q}(x, T)=\int_{b(T)}^{\infty} \frac{e^{-(x-y)^{2} /(4 T)}-e^{-(x+y-2 b(T))^{2} /(4 T)}}{\sqrt{4 \pi T}} q(y, 0) d y .
$$

Using $e^{-a}-e^{-b} \leqslant e^{-a}(b-a)$ for $0 \leqslant a \leqslant b,(x+y-2 b(T))^{2}-(x-y)^{2}=$ $4(x-b(T))(y-b(T))$, and the fact that $q(x, 0)=\delta(x)+e^{\alpha x / 2}\left[\ell e^{x}-k\right] \chi_{\left[b_{0}, 0\right]}(x)$, where $\delta$ is the Dirac measure and $\chi_{A}$ is the characteristic function of the set $A$, we then obtain, for $x \geqslant b(T)$,

$$
\begin{aligned}
q(x, T) \leqslant & \int_{b(T)}^{\infty} \frac{[x-b(T)][y-b(T)] e^{-(x-y)^{2} /(4 T)}}{\sqrt{4 \pi T^{3}}} q(y, 0) d y \\
= & \frac{(x-b(T)) e^{-x^{2} /(4 T)}}{\sqrt{4 \pi T^{3}}} \\
& \times\left\{-b(T)+\int_{b_{0}}^{0}(y-b(T))\left(\ell e^{y}-k\right) e^{-y^{2} /(4 T)+\alpha y / 2+x y /(2 T)} d y\right\} \\
\leqslant & \frac{(x-b(T)) e^{-x^{2} /(4 T)}|b(T)|}{\sqrt{4 \pi T^{3}}}\left\{1+\left|b_{0}\right|(\ell-k) e^{b^{*} b_{0} /(2 T)+\left|\alpha b_{0}\right| / 2}\right\} .
\end{aligned}
$$

Replacing $T$ by $s \geqslant 1$, we obtain

$$
p_{s}(x, s)=q(x, s) e^{-\alpha x / 2-\beta s} \leqslant|b(s)|[x-b(s)]^{+} s^{-1} \Gamma(x, s) C_{1},
$$

where $C_{1}=1+\left|b_{0}\right|(\ell-k) e^{\left(b^{*}+\alpha\right) b_{0} / 2}$. Consequently, noting that $p_{s}(x, s)=0$ for $x<b(s)$,

$$
\left\|p_{s}(\cdot, s)\right\|_{L^{\infty}(\mathbb{R})} \leqslant C_{1}\left|b^{*}\right| \max _{x>b^{*}}\left\{\left(x-b^{*}\right) s^{-1} \Gamma(x, s)\right\} \leqslant C_{2} \rho(s),
$$

where $C_{2}$ is a constant depending only on $k$ and $\ell$, and the explicit form of $\rho(s)$ follows from a direct calculation of the above maximum. In addition, for $s \geqslant 1$,

$$
\left\|p(\cdot, s)-p^{*}(\cdot)\right\|_{L^{\infty}(\mathbb{R})} \leqslant \int_{s}^{\infty}\left\|p_{s}(\cdot, t)\right\|_{L^{\infty}(\mathbb{R})} d t \leqslant \int_{s}^{\infty} C_{2} \rho(t) d t \leqslant \frac{C_{2} \rho(s)}{k} .
$$

Lower bound. Fix an arbitrary $\varepsilon>0$. Let $\underline{q}$ be the solution of

$\underline{\mathrm{q}}_{s}-\underline{\mathrm{q}}_{x x}=0$ in $[0, \infty) \times(\varepsilon, \infty), \quad \underline{\mathrm{q}}=0$ on $\{0\} \times[\varepsilon, \infty), \quad \underline{\mathrm{q}}=q$ on $[0, \infty) \times\{\varepsilon\}$.

Then by comparison, $q>\underline{q}$ on $[0, \infty) \times(\varepsilon, \infty)$. Thus, for $s>0$ and $x>0$,

$$
\begin{aligned}
q(x, s+\varepsilon) & \geqslant \mathrm{q}(x, s+\varepsilon)=\int_{0}^{\infty} \frac{e^{-(x-y)^{2} /(4 s)}-e^{-(x+y)^{2} /(4 s)}}{\sqrt{4 \pi s}} q(y, \varepsilon) d y \\
& =\frac{e^{-x^{2} /(4 s)}}{\sqrt{4 \pi s}} \int_{0}^{\infty} e^{-y^{2} /(4 s)}\left(e^{x y /(2 s)}-e^{-x y /(2 s)}\right) q(y, \varepsilon) d y .
\end{aligned}
$$


Using $e^{z}-e^{-z} \geqslant 2 z$ for every $z \geqslant 0$ we then obtain, when $x>0$ and $s>1$,

$$
q(x, s+\varepsilon) \geqslant \frac{x e^{-x^{2} /(4 s)}}{\sqrt{4 \pi s^{3}}} \int_{0}^{\infty} y q(y, \varepsilon) e^{-y^{2}} d y .
$$

Setting $C_{\varepsilon}=\int_{0}^{\infty} y e^{-y^{2}} p_{s}(y, \varepsilon) e^{\alpha y / 2} d y$ we obtain for every $x>0$ and $s>1$,

$$
\begin{aligned}
p_{s}(x, s+\varepsilon)=q(x, s+\varepsilon) e^{-\alpha x / 2-\beta(s+\varepsilon)} & \geqslant x s^{-1} \Gamma(x, s) C_{\varepsilon}, \\
p^{*}(x)-p(x, s+\varepsilon)=\int_{s}^{\infty} p_{s}(x, t+\varepsilon) d t & \geqslant \int_{s}^{s+1} x t^{-1} \Gamma(x, t) d t C_{\varepsilon} \\
& \geqslant x s^{-1} \Gamma(x, s) \int_{0}^{1} \frac{C_{\varepsilon} e^{-\beta \hat{t}}}{\sqrt{8}} d \hat{t} .
\end{aligned}
$$

Fixing $\varepsilon=1 / 2$ and finding the maximum of $x s^{-1} \Gamma(x, s)$ for $x \in(0, \infty)$ we then obtain

$$
\left\|p_{s}(\cdot, s)\right\|_{L^{\infty}(\mathbb{R})} \geqslant C_{3} \rho(s), \quad\left\|p(\cdot, s)-p^{*}(\cdot)\right\|_{L^{\infty}(\mathbb{R})} \geqslant C_{3} \rho(s),
$$

where $C_{3}$ is a positive constant depending only on $k$ and $\ell$. This completes the proof.

Proof of Theorem [3. We can derive from [3, §4]: for every $x \in \mathbb{R}$ and $s>0$,

$$
p_{s}(x, s)=\Gamma(x, s)\left\{1+I_{1}(x, s)-I_{2}(x, s)\right\},
$$

where

$$
\begin{aligned}
I_{1}(x, s) & :=\int_{b_{0}}^{0}\left[\ell e^{y}-k\right] \frac{\Gamma(x-y, s)}{\Gamma(x, s)} d y=\int_{b_{0}}^{0}\left[\ell e^{y}-k\right] e^{\alpha y / 2+x y /(2 s)-y^{2} /(4 s)}, \\
I_{2}(x, s) & :=\int_{0}^{s} \dot{b}(t)\left[\ell e^{b(t)}-k\right] \frac{\Gamma(x-b(t), s-t)}{\Gamma(x, s)} d t .
\end{aligned}
$$

Note that $I_{2}$ is positive and $I_{1}(x, s) \leqslant\left|b_{0}\right|(\ell-k) e^{\left(\alpha+b^{*}\right) b_{0} / 2}$ when $s \geqslant 1$ and $x \geqslant b^{*}$. Since we know that $p_{s}(x, s)=0$ for $x \leqslant b(s)$, we hence have the bound

$$
0 \leqslant p_{s}(x, s) \leqslant C_{1} \Gamma(x, s) \quad \forall x \in \mathbb{R}, s \geqslant 1,
$$

where the first inequality follows from the Maximum Principle.

The case $\alpha<0$. Set $\eta=(x+\alpha s) /(2 s)$. For $s \geqslant 1$,

$$
\begin{aligned}
I_{1}(x, s) & =\int_{b_{0}}^{0}\left[\ell e^{y}-k\right] e^{-y^{2} /(4 s)+\eta y} d y \\
& =e^{-\hat{y}^{2} /(4 s)} \int_{b_{0}}^{0}\left[\ell e^{y}-k\right] e^{\eta y} d y=\left[1+\frac{O(1)}{s}\right] \phi_{1}(\eta),
\end{aligned}
$$

where $\hat{y} \in\left[b_{0}, 0\right]$ and $\phi_{1} \in C^{\infty}(\mathbb{R})$ is defined by

$$
\phi_{1}(\eta)=\int_{b_{0}}^{0}\left[\ell e^{y}-k\right] e^{y \eta} d y \quad \forall \eta \in \mathbb{R} .
$$

We write $I_{2}(x, s)=\int_{0}^{s} B_{0}(s, t) e^{w} d t$, where

$$
B_{0}(s, t)=\zeta(t)\left(1-\frac{t}{s}\right)^{-\frac{1}{2}}, \quad w:=-\frac{t x^{2}}{4 s(s-t)}+\frac{x b(t)}{2(s-t)}-\frac{b^{2}(t)}{4(s-t)} .
$$


First consider the case $|\eta| \leqslant|\alpha| / 4$. Then $x / s=2 \eta-\alpha>|\alpha| / 2$, and $w \leqslant$ $-t x^{2} /\left[4 s^{2}\right] \leqslant-\alpha^{2} t / 16$. Hence, for $t>\hat{s}:=\min \left\{\frac{s}{2}, \frac{16}{\alpha^{2}} \ln s\right\}$, we have

$$
\int_{\hat{s}}^{s} B_{0}(s, t) e^{w} d t \leqslant e^{-\alpha^{2} \hat{s} / 16} \int_{0}^{s} B_{0}(s, t) d t=\frac{O(1)}{s} .
$$

When $t \in[0, \hat{s}]$,

$$
\begin{aligned}
w & =\left\{-\frac{t x^{2}}{4 s^{2}}+\frac{x b(t)}{2 s}-\frac{b^{2}(t)}{4 s}\right\}\left(1-\frac{t}{s}\right)^{-1} \\
& =-\left(\eta-\frac{\alpha}{2}\right)^{2} t+\left(\eta-\frac{\alpha}{2}\right) b(t)+O(1) \frac{t^{2}+1}{s} .
\end{aligned}
$$

Thus,

$$
\begin{aligned}
I_{2}(x, s) & =\int_{0}^{s} B_{0}(s, t) e^{w} d t=\int_{\hat{s}}^{s} B_{0}(s, t) e^{w} d t+\int_{0}^{\hat{s}} B_{0}(s, t) e^{w} d t \\
& =\frac{O(1)}{s}+\int_{0}^{\hat{s}} \zeta(t)\left[1+\frac{O(1) t}{s}\right] e^{-(\eta-\alpha / 2)^{2} t+(\eta-\alpha / 2) b(t)}\left[1+\frac{O(1)\left(t^{2}+1\right)}{s}\right] d t \\
& =\int_{0}^{\hat{s}} \zeta(t) e^{-[\eta-\alpha / 2]^{2} t+[\eta-\alpha / 2] b(t)} d t+\frac{O(1)}{s}=\phi_{2}(\eta)+\frac{O(1)}{s},
\end{aligned}
$$

where $\phi_{2}$ is defined by

$$
\phi_{2}(\eta):=\int_{0}^{\infty} \dot{b}(t)\left[\ell e^{b(t)}-k\right] e^{\left[\beta-(\eta-\alpha / 2)^{2}\right] t+\eta b(t)} d t .
$$

Since $\dot{b}(t)=O(1) t^{-3 / 2} e^{-\beta t}$ for $t \geqslant 1$ and $\dot{b}(t) d t=d b(t)$ is a bounded measure on $[0,1]$ with integrand being continuous in $t$ and analytic in $\eta, \phi_{2} \in C(\mathbb{R}) \cap$ $C^{\infty}((-\infty, \alpha / 2) \cup(\alpha / 2, \infty))$.

In summary, setting $\phi(\eta)=1+\phi_{1}(\eta)-\phi_{2}(\eta)$ we have, when $|\eta| \leqslant|\alpha| / 4$ and $s \geqslant 1$,

$$
\frac{p_{s}(x, s)}{\Gamma(x, s)}=1+I_{1}-I_{2}=\phi(\eta)+O(1) s^{-1}=\phi(0)+\phi^{\prime}(0) \eta+O(1) \eta^{2}+O(1) s^{-1} .
$$

Note that when $|\eta|>|\alpha| / 4$, both $\eta$ and $s^{-1}$ are $O(1)$, so the last expansion is still valid since it was established earlier that $p_{s} / \Gamma=O(1)$. The assertion of Theorem 3 for $p_{s} / \rho(s)$ with $\alpha<0$ thus follows with $c_{1}:=\phi(0) / \sqrt{4 \pi}$ and $c_{2}:=\phi^{\prime}(0) / \sqrt{4 \pi}$; here the positivity of $c_{1}$ follows from the inequality (2.11).

The case $\alpha \geqslant 0$. Then $k \geqslant \ell+1$, so $b_{0}=0$. Using $p_{s}(b(s), s)=0$ we obtain from (2.12) that

$$
\frac{p_{s}(x, s)}{\Gamma(x, s)}=\frac{p_{s}(x, s)}{\Gamma(x, s)}-\frac{p_{s}(b(s), s)}{\Gamma(b(s), s)}=\int_{0}^{s} B(s, t)\left\{1-e^{u}\right\} d t,
$$

where $B(s, t)$ is the same as before and

$$
\begin{aligned}
u & =\frac{x^{2}-b^{2}(s)}{4 s}-\frac{[x-b(t)]^{2}-[b(s)-b(t)]^{2}}{4(s-t)}=(x-b(s))\left\{\frac{b(t)}{2(s-t)}-\frac{t[x+b(s)]}{4 s(s-t)}\right\} \\
& =-\frac{t[x-b(s)]^{2}}{4 s(s-t)}+\frac{[x-b(s)]}{2 s}\{b(t)-t \delta(s, t)\} .
\end{aligned}
$$


Note that $u<0$ when $x+b(s) \geqslant 0$. When $|x|<-b(s), u \leqslant|b(s) \delta(s, t)|=O(1) / s$. Hence, $u \leqslant O(1) / s$ for all $x \geqslant b(s)$. Denoting $z=[x-b(s)] / \sqrt{4 s}$, we can write

$$
u=u_{1}+u_{2}, \quad u_{1}:=-\frac{t z^{2}}{s-t}, \quad u_{2}:=\frac{z}{\sqrt{s}}[b(t)-t \delta(s, t)] .
$$

We first consider the case when $z \in[0, \sqrt{s}$, i.e., $b(s)<x<b(s)+2 s$. We write $(2.13)$

$\frac{p_{s}(x, s)}{\Gamma(x, s)}=\int_{0}^{s}\left\{\left[B-B_{0}\right]\left[1-e^{u}\right]+B_{0}\left[1-e^{u_{2}}\right]+B_{0}\left[1-e^{u_{1}}\right]-B_{0}\left[1-e^{u_{2}}\right]\left[1-e^{u_{1}}\right]\right\} d t$.

Since $B-B_{0}=B_{0}\left[e^{\delta_{2}(s, t)}-1\right]=O(1) B_{0} s^{-1}$ and $u \leqslant O(1) / s$, we have for $z \geqslant 0$ and $s \geqslant 1$,

$$
\int_{0}^{s}\left[B-B_{0}\right]\left[1-e^{u}\right] d s=\frac{O(1)}{s} \int_{0}^{s} B_{0}\left|1-e^{u}\right| d t=\frac{O(1)}{s} \frac{z}{\sqrt{s}},
$$

since $\int_{0}^{s} B_{0}\left|1-e^{u}\right| d t=O(1) z / \sqrt{s}$ by the estimation below. Recalling that $\delta(s, t)=$ $O(1) s^{-1}$, we also have

$$
\begin{aligned}
\int_{0}^{s} B_{0}(s, t)\left[1-e^{u_{2}}\right] d t & =\int_{0}^{s} B_{0}(s, t)\left\{-\frac{z[b(t)+t \delta(s, t)]}{\sqrt{s}}+O(1) \frac{z^{2}}{s}\right\} d t \\
& =-\frac{z}{\sqrt{s}} \int_{0}^{\infty} \zeta(t) b(t) d t+O(1) \frac{z+z^{2}}{s}=\frac{m_{1} z}{\sqrt{s}}+O(1) \frac{z+z^{2}}{s},
\end{aligned}
$$

where $m_{1}=-\int_{0}^{\infty} b(t) \zeta(t) d t$ and the second equality is obtained by using (2.8)(2.10).

To evaluate $\int_{0}^{s} B_{0}(s, t)\left[1-e^{u_{1}}\right] d t$, we use the expansion

$$
\zeta(t)=\dot{b}(t)\left[\ell e^{b(t)}-k\right] e^{\alpha b(t) / 2+\beta t}=\hat{m} t^{-3 / 2}+O\left(t^{-2}\right),
$$

where $\hat{m}=m \beta\left[k-\ell e^{b^{*}}\right] e^{\alpha b^{*} / 2}=m_{1} / \sqrt{4 \pi}$. When $\zeta(t)$ is replaced by $\hat{m} t^{-3 / 2}$, the corresponding integral can be evaluated by the substitution $\hat{t}=-u_{1}=t z^{2} /(s-t)$, giving

$$
\int_{0}^{s} \hat{m} t^{-3 / 2}\left[1-e^{u_{1}}\right]\left(1-\frac{t}{s}\right)^{-1 / 2} d t=\frac{2 \sqrt{\pi} \hat{m} z}{\sqrt{s}}=\frac{m_{1} z}{\sqrt{s}} .
$$

We estimate the error of replacing $\zeta$ by $\hat{m} t^{-3 / 2}$ as follows. Taking $t_{1}=\max \{s / 2$, $\left.s /\left(1+z^{2}\right)\right\}$ and using $\zeta(t)=O(1) \dot{b}(t)$ for $t \in(0,1]$ and $\zeta(t)-\hat{m} t^{-3 / 2}=O(1) t^{-2}$ for $t \in[1, s]$, we obtain, when $s \geqslant 2$,

$$
\begin{gathered}
\int_{0}^{s}\left|B_{0}-\hat{m} t^{-3 / 2}\left(1-\frac{t}{s}\right)^{-1 / 2}\right|\left(1-e^{u_{1}}\right)<\int_{0}^{s}\left|\zeta-\hat{m} t^{-3 / 2}\right| \min \left\{1, \frac{t z^{2}}{s-t}\right\} \frac{\sqrt{s}}{\sqrt{s-t}} d t \\
=O(1)\left\{\int_{0}^{1}\left(t^{-3 / 2}+|\dot{b}|\right) \frac{t z^{2}}{s} d t\right. \\
\left.\quad+\int_{1}^{s / 2} \frac{z^{2}}{t s} d t+\int_{s / 2}^{t_{1}} \frac{z^{2}}{\sqrt{s} \sqrt{(s-t)^{3}}} d t+\int_{t_{1}}^{s} \frac{1}{s^{3 / 2} \sqrt{s-t}} d t\right\} \\
=O(1)\left(\frac{z^{2}}{s}+\frac{z^{2} \ln s}{s}+\frac{z^{2}}{\sqrt{s} \sqrt{s-t_{1}}}+\frac{\sqrt{s-t_{1}}}{s^{3 / 2}}\right)=O(1)\left(\frac{z}{s}+\frac{z^{2} \ln s}{s}\right) .
\end{gathered}
$$

Finally, since $\left|1-e^{u_{2}}\right|=O(1) z / \sqrt{s}$,

$$
\int_{0}^{s} B_{0}\left|\left[1-e^{u_{2}}\right]\right|\left[1-e^{u_{1}}\right] d t=\frac{O(1) z}{\sqrt{s}} \int_{0}^{s} B_{0}\left[1-e^{u_{1}}\right] d t=O(1) \frac{z^{2}}{s} .
$$


In summary, we obtain from (2.13) that when $s \geqslant 2$ and $0 \leqslant z \leqslant \sqrt{s}$ with $z=(x-b(s)) / \sqrt{4 s}$,

$\frac{p_{s}(x, t)}{\Gamma(x, s)}=\frac{z}{\sqrt{s}}\left\{2 m_{1}+\frac{O(1)}{\sqrt{s}}(1+z \ln s)\right\}=\frac{x-b(s)}{s}\left\{m_{1}+O(1)\left(s^{-1 / 2}+|x| s^{-1} \ln s\right)\right\}$.

Since $p_{s}(x, s) / \Gamma(x, s)=O(1)$, this expansion is also valid when $z>\sqrt{s}$. This implies the assertion of Theorem 3 for $p_{s} / \rho(s)$ with $c_{1}=m_{1} / \sqrt{\pi}$ for the case $\alpha=0$ and $c_{1}=m_{1} e^{\alpha b^{*} / 2} / \sqrt{4 \pi}$ for the case $\alpha>0$.

Finally, the asymptotic behavior for $p^{*}-p$ follows by integrating $p_{s}$ over $[s, \infty)$. It first requires observing that for $n>0(n=3 / 2$ for $\alpha \geq 0$ and $n=1 / 2$ for $\alpha<0)$

$$
\begin{gathered}
J:=\int_{s}^{\infty} t^{-n} e^{-x^{2} /(4 t)-\beta t}=-\int_{s}^{\infty} t^{-n} \frac{d\left(e^{-x^{2} /(4 t)-\beta t}\right)}{\beta-x^{2} /\left(4 t^{2}\right)}=\frac{s^{-n} e^{-x^{2} /(4 s)-\beta s}}{\beta-x^{2} /\left(4 s^{2}\right)}-A J, \\
A:=\frac{1}{\hat{t}}\left[\frac{n}{\beta-x^{2} /\left(4 \hat{t}^{2}\right)}+\frac{x^{2}}{2 \hat{t}^{2}} \frac{1}{\left(\beta-x^{2} /\left(4 \hat{t}^{2}\right)\right)^{2}}\right], \quad \hat{t} \in[s, \infty),
\end{gathered}
$$

which implies that for a small number $\varepsilon>0$,

$$
J=\frac{1}{1+O(1 / s)} \frac{s^{-n} e^{-x^{2} /(4 s)-\beta s}}{\beta-x^{2} /\left(4 s^{2}\right)} \quad \text { if }|x|<2 \sqrt{\beta-\varepsilon} s \text {, i.e., } \beta-\frac{x^{2}}{4 s^{2}}>\varepsilon
$$

(since $0<A=O(1 / s)$ for $\hat{t} \geq s,|x|<2 \sqrt{\beta-\varepsilon} s$ ). Then after integration and letting $s \rightarrow \infty$ for $\left(p^{*}-p\right) / \rho(s)-\Psi$, we have the assertion of the theorem with $c=c_{1} / \beta$ for $\alpha \geqslant 0$ and $c=c_{1} / k$ for $\alpha<0$. For $\alpha \geq 0$, the corresponding integration by parts produces an extra integral involving $\dot{b}(t)$. Using Theorem 1 this integral tends to zero faster than $\rho(s)$ and is assigned to the error terms. This completes the proof of Theorem 3 .

\section{REFERENCES}

[1] C. Ahn, H. Choe and K. Lee, A long time asymptotic behavior of the free boundary for an American put, Proceedings of AMS, March 30, 2009. MR2515412

[2] Xinfu Chen and J. Chadam, A mathematical analysis of the optimal boundary for American put options, SIAM J. Math. Anal., 38 (2006), 1613-1641. MR.2286022 (2007k:91131)

[3] Xinfu Chen, Huibin Cheng and J. Chadam, Non-convexity of the optimal exercise boundary for an American put option on a dividend-paying asset, preprint, submitted for publication.

Department of Mathematics, University of Pittsburgh, Pittsburgh, Pennsylvania 15260

Department of Mathematics, University of Pittsburgh, Pittsburgh, Pennsylvania 15260

Department of Mathematics, University of Pittsburgh, Pittsburgh, Pennsylvania 15260 